\title{
ARTICLE
}

\section{Population genetic structure of the South American Bryde's whale}

\author{
Estructura genética poblacional de la ballena de \\ Bryde en América del Sur
}

\section{Luis A. Pastene ${ }^{1}$, Jorge Acevedo ${ }^{2}$, Salvatore Siciliano ${ }^{3}$, Thais G.C. Sholl ${ }^{3}$, Jailson F. de Moura ${ }^{3,4}$, Paulo Henrique Ott $^{5}$ and Anelio Aguayo-Lobo ${ }^{6}$}

\author{
'Institute of Cetacean Research, Toyomi 4-5, Chuo-ku, Tokyo 104-0055, Japan \\ ${ }^{2}$ Centro de Estudios del Cuaternario Fuego-Patagonia y Antártica (Fundación CEQUA), 21 de Mayo 1690, Punta Arenas, \\ Chile.jorge.acevedo@cequa.cl; jorge.acevedo@live.cl \\ ${ }^{3}$ Escola Nacional de Saúde Pública/FIOCRUZ, Rua Leopoldo Bulhões, 1480 - 6o. andar, sala 611 \& Grupo de Estudos de \\ Mamíferos Marinhos da Região dos Lagos (GEMM-Lagos), Manguinhos, Rio de Janeiro, RJ 21041-210, Brazil \\ ${ }^{4}$ Systems Ecology, Leibniz Center for Tropical Marine Ecology (ZMT), Fahrenheitstrasse 6, 28359, Bremen, Germany \\ ${ }^{5}$ Universidade Estadual do Rio Grande do Sul (UERGS) and Grupo de Estudos de Mamíferos Aquáticos do Rio Grande do Sul \\ (GEMARS), Av. Tramandai, 976, Imbé, Rio Grande do Sul, RS 95625-000, Brazil \\ ${ }^{6}$ Instituto Antártico Chileno, Plaza Muñoz Gamero 1055, Punta Arenas, Chile
}

\begin{abstract}
Resumen.- Un análisis genético basado en secuencias de la región control del ADN mitocondrial fue realizado para investigar tanto la identidad de la especie como la estructura genética poblacional de la ballenas de Bryde Sudamericanas. El análisis genético se basó en muestras históricas, biopsias y varamientos de Chile $(n=10)$ y Brasil $(n=8)$. Para fines de comparación, secuencias publicadas de ballenas de Bryde de diferentes localidades de los océanos Índico y Pacífico (incluyendo Perú, $\mathrm{n}=24$ ) fueron incorporadas en los análisis. El análisis filogenético identificó las ballenas de Bryde de América del Sur como Balaenoptera brydei. Ninguna diferenciación genética estadísticamente significativa fue encontrada entre ballenas de Bryde de Chile y Perú. Sin embargo, fuertes diferencias genéticas se encontraron entre animales del Atlántico Sur occidental (Brasil) y Pacífico Sur oriental (Perú y Chile). También fuertes diferencias genéticas fueron encontradas entre todas las localidades de América del Sur y aquellos del Pacífico Norte occidental, Fiji y Java. Estos resultados sugieren movimiento de B. brydei en el Pacifico Sur oriental en el rango latitudinal correspondientes a Chile y Perú. Estos resultados también sugieren ningún o muy limitado movimiento de ballenas entre los océanos del Pacífico Sur y Atlántico Sur. Esto es consistente con la idea de que B. brydei no se distribuye más al sur de los 40S en ambos lados de América del Sur.
\end{abstract}

Palabras clave: Balaenoptera brydei, ADN mitocondrial, Pacífico Sur oriental, Atlántico Sur occidental

\begin{abstract}
A genetic analysis based on mitochondrial DNA control region sequences was conducted to investigate both species identity and populations genetic structure of South American Bryde's whales. The genetic analysis was based on historical, biopsy and stranding samples from Chile $(n=10)$ and Brazil $(n=8)$. For comparative purposes published sequences of the Bryde's whales from different localities of the Indian and Pacific Oceans (including Peru, $n=24$ ) were incorporated into the analysis. Results of the phylogenetic analysis identified the Bryde's whales of South America as Balaenoptera brydei. No statistically significant genetic differentiation was found between Chilean and Peruvian Bryde's whales. However, striking differences were found between western South Atlantic (Brazil) and eastern South Pacific (Peru and Chile) animals. In addition, striking genetic differences were found between all South American localities and those from the western North Pacific, Fiji and Java. These results suggest movement of B. brydei in the eastern South Pacific in the latitudinal range corresponding to Chile and Peru. These results also suggest no or very limited movement of whales between the South Pacific and the South Atlantic Oceans. This is consistent with the notion that B. brydei is not distributed further south of approximately 40 오 on both sides of South America.
\end{abstract}

Key words: Balaenoptera brydei, mitochondrial DNA, eastern South Pacific, western South Atlantic

\section{INTRODUCTION}

Bryde's whales have been difficult to study in several regions of the world due to its similarity with the sei whale Balaenoptera borealis (Lesson, 1828). Whaling stations in the eastern South Pacific continued to confuse both species, in Peru until 1973 and in Chile until the end of commercial whaling in 1983 (Valdivia et al. 1981, Gallardo et al. 1983). In Brazil, this confusion remained until 1967 at some stations, and until the end of the commercial 
whaling period (1988) in others (Zerbini et al. 1997). In the North Pacific, Bryde's and sei whale catches were reported separately at a much earlier date, in the mid1950s.

In the eastern South Pacific, analyses based on sighting and catch distribution suggested that Bryde's whales are distributed in Peruvian waters almost throughout the year with abundance changing seasonally (Pastene \& Ohsumi 1998) ${ }^{1}$. The first record of Bryde's whale in Chile was reported by Clarke \& Aguayo (1965) on the basis of an individual caught off Iquique $\left(20^{\circ} \mathrm{S}\right)$. Subsequently Gallardo et al. (1983) reported the occurrence of Bryde's whales based on sighting data between $32^{\circ}-36^{\circ} \mathrm{S}$ approximately. Pastene et al. $(1983)^{2}$ confirmed the occurrence of Bryde's whales in central Chile through the examination of three animals caught in the

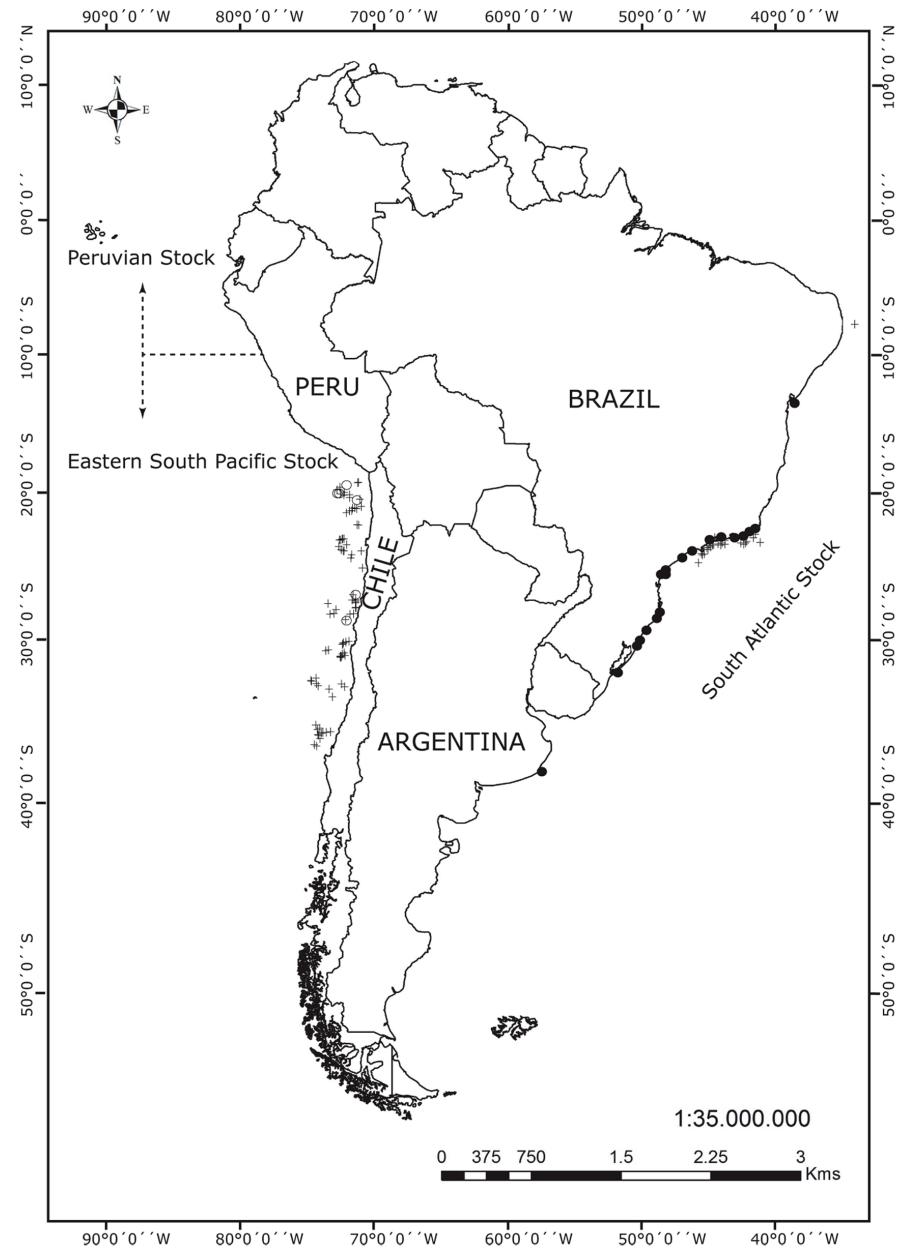

latitudinal range of $33^{\circ}-35^{\circ} \mathrm{S}$. Aguayo et al. (1998) reported a single sighting of a Bryde's whale in the adjacent waters of San Felix and San Ambrosio Islands (2659'S; 86³9'W) on 3 September 1994. Currently, the distribution of Bryde's whales in the eastern South Pacific off Chile based on animals confirmed as Bryde's whales and 'like' Bryde's whales (Fig. 1) show a continuity in geographical distribution between the latitudinal range of $18^{\circ} 43^{\prime} \mathrm{S}$ and $37^{\circ} 58^{\prime} \mathrm{S}$ and in the longitudinal range between the Chilean coast and $76^{\circ} 16^{\prime} \mathrm{W}$. Moreover, seasonal pattern of occurrence of Bryde's whales in the eastern South Pacific was consistent with the hypothesis of a north (Peru)-south (to latitude $38^{\circ} \mathrm{S}$ in Chile) movement of whales in the eastern South Pacific in spring and summer (Pastene 1982).

Figure 1. Distribution of sightings ( + ), like Bryde's (o) and strandings $(\bullet)$ of Bryde's whale in Chilean and Brazilian waters. Sources: Clarke \& Aguayo (1965), Gallardo et al. (1983), Pastene et al. (1983), Zerbini et al. (1997), Aguayo et al. (1998), Findlay et al. (1998), Siciliano et al. (2004), Andriolo et al. (2010), Figuereido et al. (2014) and unpublished data / Distribución de los avistamientos (+), probable Bryde (o) y varamientos $(\bullet)$ de ballenas de Bryde en aguas de Chile y Brazil. Fuentes: Clarke \& Aguayo (1965), Gallardo et al. (1983), Pastene et al. (1983), Zerbini et al. (1997), Aguayo et al. (1998), Findlay et al. (1998), Siciliano et al. (2004), Andriolo et al. (2010), Figueiredo et al. (2014) y datos no publicados

${ }^{1}$ Pastene LA \& S Ohsumi. 1998. A brief review of the information on distribution and stock identity of Bryde's whales (Balaenoptera edeni) in the eastern South Pacific. Paper SC/50/CAWS6 presented to the IWC Scientific Committee, June 1998 (unpublished), 18 pp. 2Pastene LA, M Acevedo \& VA Gallardo. 1983. A note on Chilean Bryde's whales. Paper SC/35/Ba4 presented to the IWC Scientific Committee June 1983 (unpublished), 9 pp. 
In the western South Atlantic, analysis of sighting and stranding data suggested that Bryde's whales occur regularly in coastal areas mainly in southeastern and southern Brazil (Fig. 1). Sighting of this species were recorded mainly in summer and autumn while strandings have been recorded through the year (Zerbini et al. 1997, Siciliano et al. 2004). Bastida \& Rodriguez (2009) reported that in the Atlantic coastal waters of South America, the southernmost records corresponded to a couple of strandings recorded on the northern coasts of Argentina

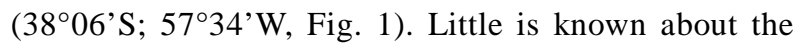
relationship between western South Atlantic and eastern South Pacific Bryde's whales.

The taxonomy of Bryde's whales is still unresolved. Some authors (e.g., Wada et al. 2003) recognize two species, the smaller one B. edeni Anderson, 1879 (Eden's whale) and a larger one B. brydei Olsen, 1913 (Bryde's whale), while others (e.g., Kershaw et al. 2013) assign these species a sub-specific status: $B$. edeni edeni and $B$. edeni brydei, respectively. The smaller one inhabits primarily coastal and continental shelf waters of the Northern Indian Ocean and the western Pacific Ocean while the larger one inhabits tropical and warm temperature waters worldwide (Rice 1998). Wada et al. (2003) provided genetics, external morphology and osteology evidences to separate $B$. bryde $i$ and $B$. eden $i$ into two distinct species. The present study follows the taxonomic classification of Wada et al. (2003).

Peru, Chile and Brazil were involved in commercial whaling of Bryde's whales until approximately the mid 1980's. The International Whaling Commission's Scientific Committee (IWC-SC) defined the geographic boundaries of stocks for management purposes: a 'Peruvian stock' from $10^{\circ} \mathrm{N}-10^{\circ} \mathrm{S}$ and from the South American coast to $110^{\circ} \mathrm{W}$, including the Galápagos Archipelago; and an 'Eastern South Pacific stock' from the South American coast and $150^{\circ} \mathrm{W}$, excluding the Peruvian stock area (Donovan 1991). Whales caught in Brazil were from the 'South Atlantic stock' (Donovan 1991). There is, however, limited biological evidence to support those geographic delineations.

The objective of this study were, firstly to examine the species identity and secondly to elucidate the population genetic structure of the species around South America, through the genetic analyses of samples collected on both sides of South America. Given the known geographical distribution of $B$. edeni and $B$. brydei (Rice 1998), our expectation was that the species distributed in South American waters correspond to the latter one. Within the species, our expectation was that the genetic composition of whales from Peru and Chile is similar based on the hypothesised seasonal movement in the eastern South Pacific (Pastene 1982), and that whales from the eastern South Pacific and western South Atlantic are genetically distinct given that their distribution is limited to regions north of $40^{\circ} \mathrm{S}$ and therefore no or only limited interchange of whales occurs between those ocean basins due to the landmass barrier (southern South America) that extends south to $53^{\circ} \mathrm{S}$.

\section{Materials AND METHODS}

\section{SAMPLES}

Biopsy samples from the Chilean Bryde's whales were obtained by the IWC Southern Ocean Whale and Ecosystem Research Program (IWC-SOWER) survey conducted in December 1997 (Findlay et al. 1998) $(n=8)$. Biopsy skin samples were collected using the Paxarm system (Krützen et al. 2002) and crossbows. In addition baleen plates were available from whales caught in Chile in April $1983(n=2)$. Muscle samples from the Brazilian Bryde's whales were obtained from stranded animals along the southern and southeastern coast of Brazil in January, February, March, August, September and October between 2004 and $2010(n=8)$. Skin/muscle samples were stored in ethanol $100 \%$ until their use. Sequences from the Peruvian Bryde's whales published in Kanda et al. (2007) were obtained from muscle samples collected during a former Japanese coastal whaling operation in Peru in January-March 1983. Samples were stored at $-20^{\circ} \mathrm{C}$ until their use. Figure 2 shows the geographic distribution of the samples used in the present study and Table 1 shows ancillary information of the sampling in Chile and Brazil.

\section{LABORATORY ANALYSIS}

Genomic DNA was extracted from approximately $0.05 \mathrm{~g}$ of the outer epidermal layer of the skin or muscle using the protocol of Sambrook \& Russell (2001). In the case of baleen plates, DNA was extracted using the Bio 101 'Genclean Kit for Ancient DNA'. Extracted DNAs were stored in TE buffer (10 mM Tris-HCl, 1 mM EDTA, pH 8.0).

${ }^{3}$ Findlay K, R Pitman, T Tsurui, K Sakai, P Ensor, H Iwakami, D Ljungblad, H Shimada, D Thiele, K Van Waerebeek, R Hucke-Gaete \& GP Sanino-Vatier. 1998. 1997/1998 IWC-Southern Ocean Whale and Ecosystem Research (IWC-SOWER) Blue Whale Cruise, Chile. Paper SC/SO/Rep.2 presented to the IWC Scientific Committee (unpublished), 40 pp. 


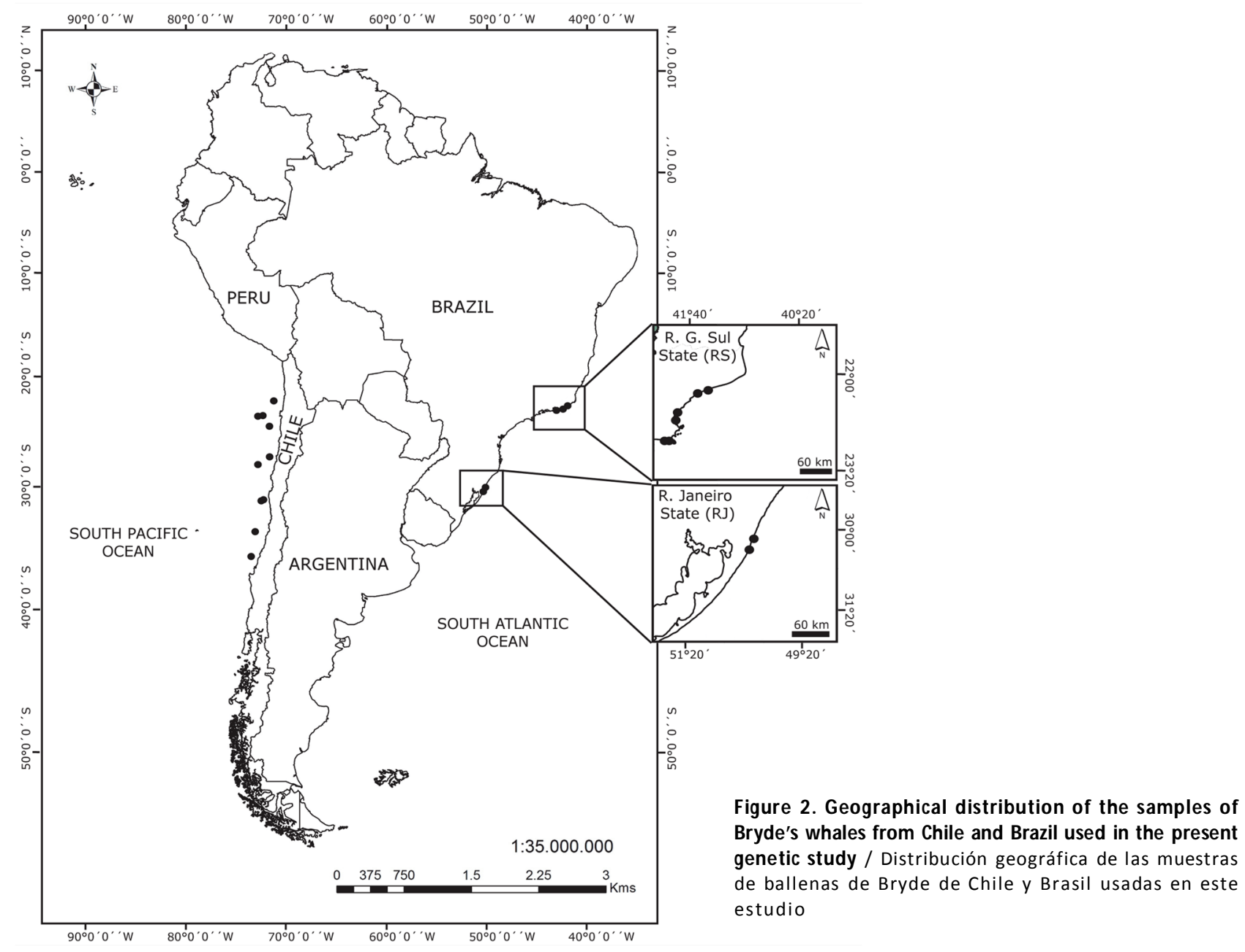

Sequencing analyses of the 299 base pairs (bp) control region of mitochondrial DNA (mtDNA) was conducted using the primers MT4 (Arnason et al. 1993) and P2 (Kanda et al. 2007). Reactions were carried out in $50 \mathrm{uL}$ volumes containing $100 \mathrm{mM} \mathrm{KCl}, 20 \mathrm{mM}$ Tris-HCl, $0.1 \mathrm{mM}$ EDTA, $1 \mathrm{mM}$ DTT, $0.5 \%$ Tween 20, 0.5\% Nonidet P-40, $200 \mathrm{uM}$ dNTPs, $2.5 \mathrm{pM}$ of each oligo-nucleotide and one unit of Taq DNA polymerase (TaKaRa Ex Taq). After an initial denaturation step at $95^{\circ} \mathrm{C}$ for $5 \mathrm{~min}$, a PCR amplification cycle of $30 \mathrm{~s}$ at $94^{\circ} \mathrm{C}$, followed by $30 \mathrm{~s}$ at $50^{\circ} \mathrm{C}$ and $30 \mathrm{~s}$ at $72^{\circ} \mathrm{C}$ was repeated 30 times. The amplification was completed with a final extension step of $10 \mathrm{~min}$ at $72^{\circ} \mathrm{C}$. Subsequent cycle sequencing reactions were performed with $100 \mathrm{ng}$ of products generated in the above PCR amplifications using the Prism ${ }^{\mathrm{TM}} \mathrm{dRhodamine}$ Terminator
Cycle Sequencing Kit (Applied Biosystems, Inc.). The oligo-nucleotides used to prime the cycle sequencing reaction were the same as those used in the initial PCR amplification. A total of 25 cycles with $10 \mathrm{~s}$ at $96^{\circ} \mathrm{C}, 20 \mathrm{~s}$ at $56^{\circ} \mathrm{C}$ and $4 \mathrm{~min}$ at $60^{\circ} \mathrm{C}$ were performed. Purification of cycle sequencing reactions was made using AutoSeq G50 Dye Terminator Removal Kit. The nucleotide sequence of each cycle sequencing reaction was determined by electrophoresis through a 5\% Long Ranger ${ }^{\mathrm{TM}}$ (FMC, Inc.) denaturing polyacrylamide matrix on a DNA Prism ${ }^{\mathrm{TM}} 377$ DNA Sequencer (Applied Biosystems, Inc.) under standard conditions. Both DNA strand were sequenced for each sample. A consensus sequence was created for each sample after resolving potential disagreements in aligned sequences. 
Table 1. Samples of the Bryde's whales from Chilean and Brazilian waters used in the present analyses / Muestras de ballenas de Bryde de Chile y Brazil utilizadas en el análisis

\begin{tabular}{|c|c|c|c|c|c|}
\hline Location & $\begin{array}{c}\text { Sampling } \\
\text { date }\end{array}$ & Source & $\begin{array}{l}\text { Latitude } \\
\text { (S) }\end{array}$ & $\begin{array}{l}\text { Longitude } \\
\text { (W) }\end{array}$ & Reference \\
\hline Chilean waters & 10 Apr 1983 & Catch & $33^{\circ} 55^{\prime}$ & $72^{\circ} 46^{\prime}$ & Pastene (1983) \\
\hline Chilean waters & 12 Apr 1983 & Catch & $35^{\circ} 19^{\prime}$ & $73^{\circ} 09^{\prime}$ & Pastene (1983) \\
\hline Chilean waters & 14 Dec 1997 & Biopsy & $24^{\circ} 08^{\prime}$ & $71^{\circ} 45^{\prime}$ & Findlay et al. (1998) \\
\hline Chilean waters & 19 Dec 1997 & Biopsy & $21^{\circ} 46^{\prime}$ & $71^{\circ} 15^{\prime}$ & Findlay et al. (1998) \\
\hline Chilean waters & 21 Dec 1997 & Biopsy & $22^{\circ} 47^{\prime}$ & $72^{\circ} 25^{\prime}$ & Findlay et al. (1998) \\
\hline Chilean waters & 21 Dec 1997 & Biopsy & $22^{\circ} 50^{\prime}$ & $72^{\circ} 39^{\prime}$ & Findlay et al. (1998) \\
\hline Chilean waters & 24 Dec 1997 & Biopsy & $27^{\circ} 02^{\prime}$ & $71^{\circ} 38^{\prime}$ & Findlay et al. (1998) \\
\hline Chilean waters & 26 Dec 1997 & Biopsy & $27^{\circ} 41^{\prime}$ & $72^{\circ} 54^{\prime}$ & Findlay et al. (1998) \\
\hline Chilean waters & 30 Dec 1997 & Biopsy & $30^{\circ} 53^{\prime}$ & $72^{\circ} 32^{\prime}$ & Findlay et al. (1998) \\
\hline Chilean waters & 30 Dec 1997 & Biopsy & $30^{\circ} 49^{\prime}$ & $72^{\circ} 28^{\prime}$ & Findlay et al. (1998) \\
\hline Brazilian waters & 26 Feb 2004 & Stranding & $22^{\circ} 14^{\prime}$ & $41^{\circ} 32^{\prime}$ & This study \\
\hline Brazilian waters & 23 Jan 2005 & Stranding & $22^{\circ} 33^{\prime}$ & $41^{\circ} 58^{\prime}$ & This study \\
\hline Brazilian waters & 28 Sep 2006 & Stranding & $22^{\circ} 40^{\prime}$ & $41^{\circ} 59^{\prime}$ & This study \\
\hline Brazilian waters & 18 Aug 2007 & Stranding & $22^{\circ} 58^{\prime}$ & $42^{\circ} 02^{\prime}$ & This study \\
\hline Brazilian waters & 11 Oct 2008 & Stranding & $22^{\circ} 57^{\prime}$ & $42^{\circ} 05^{\prime}$ & This study \\
\hline Brazilian waters & 08 Mar 2010 & Stranding & $22^{\circ} 17^{\prime}$ & $41^{\circ} 40^{\prime}$ & This study \\
\hline Brazilian waters & 20 Jan 2005 & Stranding & $30^{\circ} 13^{\prime}$ & $50^{\circ} 13^{\prime}$ & This study \\
\hline Brazilian waters & 21 Jan 2005 & Stranding & $30^{\circ} 20^{\prime}$ & $50^{\circ} 16^{\prime}$ & This study \\
\hline
\end{tabular}

\section{AnALysis OF GENETIC DATA}

\section{Phylogenetic analysis}

Sequences were aligned by eye using Sequence Navigator (Applied Biosystem, Inc.). Variable sites and unique sequences (haplotypes) were identified using the program MacClade (Maddison \& Maddison 2000).

A phylogenetic tree of haplotypes was generated using the Neighbor-Joining method (Saitou \& Nei 1987) as implemented in the program PHYLIP (Felsenstein 1993). Genetic distances among haplotypes were estimated using the program DNADIST of PHYLIP, based on Kimura's 2-parameter model (Kimura 1980). A transitiontransversion ratio of 5:1 was used. For comparative purposes, mtDNA control region sequences of $B$. edeni published by Yoshida \& Kato (1999) and Wada et al. (2003) and of B. brydei published by Kanda et al. (2007), were included in the estimation. The genealogy was rooted using the homologous sequence from $B$. omurai and $B$. borealis published in Wada et al. (2003). To estimate support for each node a total of 1,000 bootstrap simulations were conducted and the majority-rule consensus genealogy estimated. The genealogy of mtDNA haplotypes was also inferred using the maximum likelihood (ML) method as implemented in the program MEGA6 (Tamura et al. 2013). The Tamura-Nei's threeparameter substitution model with Gamma distribution (Tamura \& Nei 1993) was set as substitution model to reconstruct the ML tree based on the results of the ModelTest. B. omurai and B. borealis were used as outgroups. Support for the groupings was estimated with 1,000 bootstrap replications.

\section{GENETIC DIVERSITY AND POPULATION DIFFERENTIATION}

The degree of mtDNA diversity within each area was estimated using the nucleotide diversity (Nei \& Li 1979). The level of differentiation of mtDNA between populations was estimated using net interpopulation genetic distance, dA (Nei 1987). Heterogeneity tests among whales from different oceanic regions were conducted as described in Hudson et al. (1992), using the chi-square, the $H s t$ and the $K s t^{*}$ statistics. The level of statistical significance was estimated from 10,000 Monte Carlo simulations as the proportion of simulations in which a similar or more extreme value of chi-square, $H s t$ 
Table 2. Frequencies of haplotypes in B. brydei by geographical locality (WNP= western North Pacific). Haplotypes were numbered following Kanda et al. (2007). Haplotypes 52-55 are new haplotypes with regard those in Kanda et al. (2007) / Frecuencias de haplotipos en B. brydei por localidad geográfica. (WNP= Pacífico Noroccidental). Haplotipos fueron numeradas siguiendo a Kanda et al. (2007). Haplotipos 52-55 son nuevos en relación a aquellos de Kanda et al. (2007)

\begin{tabular}{|c|c|c|c|c|c|c|c|}
\hline Haplotype & WNP & FIJI & PERU & Chile & Brazil & JAVA & Total \\
\hline 1 & 50 & 15 & 0 & 0 & 0 & 0 & 65 \\
\hline 2 & 2 & 0 & 0 & 0 & 0 & 0 & 2 \\
\hline 3 & 35 & 0 & 1 & 0 & 0 & 0 & 36 \\
\hline 4 & 3 & 0 & 0 & 0 & 0 & 0 & 3 \\
\hline 5 & 156 & 3 & 1 & 0 & 0 & 0 & 160 \\
\hline 6 & 8 & 0 & 0 & 0 & 0 & 0 & 8 \\
\hline 7 & 14 & 0 & 0 & 0 & 0 & 0 & 14 \\
\hline 8 & 14 & 0 & 1 & 0 & 0 & 0 & 15 \\
\hline 9 & 3 & 0 & 0 & 0 & 0 & 0 & 3 \\
\hline 10 & 6 & 0 & 0 & 0 & 0 & 0 & 6 \\
\hline 11 & 5 & 0 & 0 & 0 & 0 & 0 & 5 \\
\hline 12 & 4 & 0 & 0 & 0 & 0 & 0 & 4 \\
\hline 13 & 5 & 0 & 0 & 0 & 0 & 0 & 5 \\
\hline 14 & 1 & 0 & 0 & 0 & 0 & 0 & 1 \\
\hline 15 & 8 & 0 & 0 & 0 & 0 & 0 & 8 \\
\hline 16 & 6 & 0 & 0 & 0 & 0 & 0 & 6 \\
\hline 17 & 2 & 0 & 1 & 0 & 0 & 0 & 3 \\
\hline 18 & 14 & 0 & 0 & 0 & 0 & 0 & 14 \\
\hline 19 & 8 & 0 & 0 & 0 & 0 & 0 & 8 \\
\hline 20 & 4 & 0 & 0 & 0 & 0 & 0 & 4 \\
\hline 21 & 3 & 0 & 0 & 0 & 0 & 0 & 3 \\
\hline 22 & 5 & 0 & 0 & 0 & 0 & 0 & 5 \\
\hline 23 & 2 & 0 & 0 & 0 & 0 & 0 & 2 \\
\hline 24 & 2 & 0 & 0 & 0 & 0 & 0 & 2 \\
\hline 25 & 7 & 0 & 0 & 0 & 0 & 0 & 7 \\
\hline 26 & 7 & 0 & 0 & 0 & 0 & 0 & 7 \\
\hline 27 & 1 & 0 & 0 & 0 & 0 & 0 & 1 \\
\hline 28 & 6 & 0 & 0 & 0 & 0 & 0 & 6 \\
\hline 29 & 8 & 0 & 0 & 0 & 0 & 0 & 8 \\
\hline 30 & 2 & 0 & 0 & 0 & 0 & 0 & 2 \\
\hline 31 & 1 & 0 & 0 & 0 & 0 & 0 & 1 \\
\hline 32 & 1 & 0 & 0 & 0 & 0 & 0 & 1 \\
\hline 33 & 3 & 0 & 0 & 0 & 0 & 0 & 3 \\
\hline 34 & 2 & 0 & 0 & 0 & 0 & 0 & 2 \\
\hline 35 & 1 & 0 & 0 & 0 & 0 & 0 & 1 \\
\hline 36 & 2 & 3 & 1 & 1 & 7 & 0 & 14 \\
\hline 37 & 0 & 2 & 0 & 0 & 0 & 0 & 2 \\
\hline 38 & 0 & 1 & 0 & 0 & 0 & 0 & 1 \\
\hline 39 & 0 & 0 & 1 & 0 & 0 & 0 & 1 \\
\hline 40 & 0 & 0 & 1 & 0 & 0 & 0 & 1 \\
\hline 41 & 0 & 0 & 1 & 0 & 0 & 0 & 1 \\
\hline 42 & 0 & 0 & 5 & 3 & 0 & 0 & 8 \\
\hline 43 & 0 & 0 & 1 & 0 & 0 & 0 & 1 \\
\hline 44 & 0 & 0 & 1 & 0 & 0 & 0 & 1 \\
\hline 45 & 0 & 0 & 3 & 2 & 0 & 0 & 5 \\
\hline 46 & 0 & 0 & 5 & 1 & 0 & 0 & 6 \\
\hline 47 & 0 & 0 & 1 & 0 & 0 & 0 & 1 \\
\hline 48 & 0 & 0 & 0 & 0 & 0 & 19 & 19 \\
\hline 49 & 0 & 0 & 0 & 0 & 0 & 2 & 2 \\
\hline 50 & 0 & 0 & 0 & 0 & 0 & 1 & 1 \\
\hline 51 & 0 & 0 & 0 & 0 & 0 & 1 & 1 \\
\hline 52 & 0 & 0 & 0 & 1 & 0 & 0 & 1 \\
\hline 53 & 0 & 0 & 0 & 1 & 0 & 0 & 1 \\
\hline 54 & 0 & 0 & 0 & 1 & 0 & 0 & 1 \\
\hline 55 & 0 & 0 & 0 & 0 & 1 & 0 & 1 \\
\hline Total & 401 & 24 & 24 & 10 & 8 & 23 & 490 \\
\hline
\end{tabular}

or $K s t^{*}$ was observed. For the analyses of mtDNA diversity and population differentiation, sequences from B. brydei from the eastern South Pacific, western North Pacific, western South Pacific and eastern Indian Ocean published by Kanda et al. (2007) were used for comparative purposes.

\section{Results}

DNA was successfully extracted and sequenced from the ten and eight samples from Chilean and Brazilian waters, respectively. The final data set included the first 299 nucleotides of the mtDNA control region from a total of 490 specimens (including the 18 from Chile and Brazil and those examined by Kanda et al. 2007). There were 38 segregating sites discriminating a total of 55 haplotypes in the total sample (Fig. 3 and Table 2). Haplotypes were numbered following Kanda et al. (2007) (Br01, Br02, etc.). All mutations were transitions. The additional 18 samples from Chile and Brazil discriminated four new haplotypes in relation to the data set used by Kanda et al. (2007): haplotypes '52', '53', '54' and '55' (Fig. 3 and Table 2). The sequences of these four haplotypes have been deposited in GenBank under Accession numbers KT191131, KT191132, KT191133 and KT191134, respectively.

Figure 3 includes three haplotypes of $B$. edeni, one from $B$. omurai and one from B. borealis (Yoshida \& Kato 1999, Wada et al. 2003). Fixed differences in the nucleotide sequence between the 55 haplotypes $(n=490)$ and $B$. edeni were observed at eleven nucleotide positions (positions 28, 45, 46, 58, 77, 82, 84, 149, 190, 194, and 237, see Fig. 3).

\section{Phylogenetic analysis}

The haplotypes of South American whales clustered together Kanda et al. (2007)'s B. brydei haplotypes. The 55 ' $\mathrm{Br}$ ' haplotypes were clearly separated from $B$. edeni, B. omurai and B. borealis (Fig. 4), a pattern supported by high bootstrap values. These haplotypes were associated with Wada et al. (2003)'s B. brydei. B. edeni and B. omurai are closer related with $B$. borealis than with $B$. brydei, and this pattern was also supported by high bootstrap values. Within B. brydei, three main clades were observed but none was supported by high bootstrap values, and none was geographic-specific. A similar phylogenetic pattern was found by the ML method (Fig. 5). 


\begin{tabular}{|c|c|c|c|c|c|c|c|c|}
\hline & 10 & 20 & 30 & 40 & 50 & 60 & 70 & \\
\hline & & & & & 11111111 & 1111222222 & 2222222222 & 22222222 \\
\hline & 1111222 & 2333333444 & 4455555566 & 7778888899 & 9923334445 & 6999000001 & 1122223333 & 33455578 \\
\hline & 1790179018 & 9014789035 & 6734568903 & 3791234934 & 6784890590 & 3046356783 & 4536890234 & 78002333 \\
\hline $\mathrm{Br} 01$ & СТАТСТАСТT & TTGCGTATAC & -TTAACT--T & TTAGGGGAAT & --TTAGCACT & TTATAAGCAT & CATCCAGCGA & ACCTTTAT \\
\hline $\mathrm{BrO} 2$ & $\ldots G \ldots \ldots$ & $\ldots \ldots \ldots$ & $\ldots \ldots \ldots$ & $\ldots \ldots \ldots \ldots$ & TA........ & $\ldots \ldots \ldots \ldots$ & . СTT...A. & $\ldots \ldots \mathrm{G}$. \\
\hline $\mathrm{Br} 03$ & $\ldots G \ldots \ldots$ & $\ldots \ldots \ldots$ & $\ldots \ldots \ldots \ldots$ & $\ldots \ldots \ldots \ldots$ & TA....... & $\ldots \ldots \ldots$ G. & ...TG.A. & ....CG. \\
\hline $\mathrm{Br} 04$ & $\ldots G \ldots \ldots$ & $\ldots \ldots \ldots$ & $\ldots \ldots \ldots$ & $\ldots \ldots \ldots$ & $\ldots \ldots$ & C.....G. & ...TG.TA. & $\ldots \ldots$ \\
\hline Br05 & $\ldots G \ldots \ldots$ & $\ldots \ldots \ldots$ & $\ldots \ldots \ldots$ & $\ldots \ldots \ldots$ & $\ldots \ldots \ldots$ & C......G. & ....TG.TAG & $\ldots \ldots$ \\
\hline Br06 & $\ldots G \ldots \ldots$ & $\ldots$ & $\ldots \ldots \ldots$ & $\ldots \ldots \ldots \ldots$ & $\ldots \ldots \ldots$ & C......G. & ...TG.TA. &. $\mathrm{T} \ldots \mathrm{C}$ \\
\hline $\mathrm{Br} 07$ & $\ldots \ldots \ldots$ & $\ldots$ & $\ldots \ldots \ldots$ & $\ldots \ldots \ldots$ & $\ldots \ldots \ldots$ & $\ldots \ldots \ldots$ & $\ldots \ldots \ldots$ & ...... \\
\hline$=08$ & $\ldots G \ldots \ldots$ & $\ldots \ldots \ldots$ & $\ldots \ldots \ldots$ & $\ldots \ldots \ldots$ & $\ldots \ldots$ & C......G. & .... TG.TAG & $\ldots$.... \\
\hline 209 & $\ldots G \ldots \ldots$ & $\ldots \ldots$ & $\ldots \ldots \ldots$ & 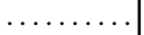 & $\ldots \ldots$ & C......G. & ...TG.A. & . T... \\
\hline$=10$ & $\ldots G \ldots \ldots$ & $\ldots \ldots \ldots$ & $\ldots \ldots \ldots$ & $\ldots \ldots \ldots$ & $\ldots \ldots \ldots \ldots$ & C....... & ...TG.TA & . т.... \\
\hline$=11$ & $\ldots G \ldots \ldots$ & $\ldots \ldots \ldots$ & $\ldots \ldots \ldots \ldots$ & $\ldots \ldots \ldots \ldots$ & $\ldots \ldots \ldots$ & C.....G. & . . TTG.TA. & .T... C \\
\hline$r 12$ & $\ldots G \ldots \ldots$ & $\ldots \ldots \ldots$ & $\ldots \ldots \ldots$ & $\ldots \ldots \ldots$ & $\ldots \ldots \ldots$ & $\ldots \ldots \mathrm{TG}$ & ...TG. A. & $\ldots \ldots$ \\
\hline Br13 & $\ldots G \ldots \ldots$ & $\ldots \ldots \ldots$ & $\ldots \ldots \ldots$ & $\ldots \ldots \ldots$ & $\ldots \ldots \ldots$ & C....A.G. & ...TG.TA. & $\ldots \ldots$ \\
\hline $\mathrm{Br} 14$ & $\ldots G \ldots \ldots$ & ........... & $\ldots \ldots \ldots$ & $\ldots \ldots \ldots \ldots$ & $\ldots \ldots$ G. & C....... & ...TG.TA & $\ldots \ldots$ \\
\hline $\operatorname{Br} 15$ & $\ldots G \ldots \ldots$ & $\ldots \ldots \ldots$ & $\ldots \ldots \ldots$ & $\ldots \ldots \ldots$ & $\ldots \ldots \ldots$ & $\ldots \ldots \mathrm{TG}$ & $\ldots$ TG $\ldots$ & $\ldots \ldots$ \\
\hline$r 16$ & $\ldots$ G.T.... & $\ldots \ldots$ & $\ldots \ldots \ldots$ & $\ldots \ldots \ldots$ & $\ldots \ldots \ldots$ & C....... & ....TG.TA. &. $\mathrm{T} \ldots$. \\
\hline $\operatorname{Br} 17$ & $\ldots G \ldots \ldots$ & $\ldots \ldots$ & $\ldots \ldots \ldots$ & $\ldots \ldots \ldots$ & $\ldots \ldots \ldots \ldots$ & $\ldots \ldots \ldots G$ & $\ldots$ TG. A & . т.... \\
\hline $\operatorname{Br} 18$ & $\ldots G \ldots \ldots$ & & & & TA....... & $\ldots \ldots \ldots$ & ...TT... A. & $\ldots \ldots G$. \\
\hline $\operatorname{Br} 19$ & $\ldots G \ldots \ldots$ & $\ldots$ & $\ldots$ & $\ldots$ & $\ldots$. G.... & C....... & ...TG.TA. & $\ldots \ldots$ \\
\hline $\mathrm{Br} 20$ & $\ldots G \ldots \ldots$ & & & & TA....... & $\ldots \ldots \ldots$ & ...TG.A. & $\ldots \ldots G$. \\
\hline $\mathrm{Br} 21$ & $\ldots G C \ldots \ldots$ & $\ldots \ldots$ & $\ldots \ldots \ldots$ & & $\ldots \ldots$ & C.....G. & ...TG.TA. & $\ldots c \ldots$ \\
\hline$r 22$ & $\ldots G \ldots \ldots$ & & $\ldots \ldots$ & & & c........ & ....TG.TA. & . т.... \\
\hline 23 & $\ldots G \ldots \ldots$ & $\cdots$ & $\ldots \ldots \ldots$ & $\ldots \ldots \ldots$ & $\ldots \ldots \ldots$ & $\ldots c \ldots \ldots$ & ...TG.A. & .тт.... \\
\hline 24 & . G.... & $\ldots \ldots \mathrm{T}$ & $\ldots \ldots \ldots c$ & $\ldots \ldots \ldots G$ & TA.....G. & ....G.T. & ....TG. .A. & $\ldots \ldots$ \\
\hline 25 & $\ldots G \ldots \ldots$ & $\ldots \ldots \ldots$ &.$c \ldots \ldots$ & $\ldots \ldots \ldots \ldots$ & $\ldots \ldots \ldots \ldots$ & C......G. & ...TG.TA. & $\ldots \ldots \ldots$ \\
\hline 26 & $\ldots G \ldots \ldots$ & $\ldots \ldots \ldots$ & $\ldots \ldots \ldots$ & $\ldots \ldots \ldots$ & $\ldots \ldots \ldots$ & $c \ldots \ldots$ & $\ldots \mathrm{T}$ & $\ldots c \ldots$ \\
\hline 27 & $\ldots G \ldots \ldots$ & $c \ldots \ldots$ & $\ldots \ldots \ldots$ & $\ldots \ldots \ldots \ldots$ & $\ldots$...... & C...... & ...TG.TA. & $\ldots \ldots \ldots$ \\
\hline$=28$ & $\ldots G \ldots \ldots$ & $\ldots \ldots \ldots$ & $\ldots \ldots \ldots$ & $\ldots \ldots \ldots \ldots$ & $\ldots \ldots \ldots$ & C......G. & ....TG.TAG & . T.... \\
\hline 229 & $\ldots G \ldots \ldots$ & $\ldots \ldots \ldots$ & $\ldots \ldots \ldots$ & $\ldots \ldots \ldots$ & $\ldots \ldots \ldots$ & C....... & ....TG.TA & $\ldots c \ldots$ \\
\hline$=30$ & $\ldots$ G...T. & $\ldots \ldots \ldots$ & $\ldots \ldots \ldots$ & $\ldots \ldots \ldots$ & TA.C...G. & $\ldots \ldots \ldots$ G. & T...TG..A. & $\ldots \ldots \ldots$ \\
\hline$=31$ & $\ldots G \ldots \ldots$ & & & $\ldots$ & & C......G. & ...TTG..A. & . T.... \\
\hline Br32 & $\ldots G \ldots \ldots$ & $\cdots$ & $\ldots$ & $\ldots \ldots$ & & C......G. & ...T. . TA & . т.... \\
\hline $\mathrm{Br} 33$ & $\ldots G \ldots \ldots$ & $\ldots$ & $\ldots \ldots \ldots$ & $\ldots \ldots \ldots$ & $\ldots \ldots \ldots \ldots$ & C....... & T...TG.TAG & $\ldots$.... \\
\hline $\operatorname{Br} 34$ & $\ldots G \ldots T \ldots$ & $\ldots \ldots \ldots \mathrm{T}$ & $\ldots \ldots \ldots c$ & $\ldots \ldots \ldots G$ & TA....G. & $\ldots \ldots \ldots$ & $\ldots$ TG.A. & ....... \\
\hline Br35 & $\ldots G \ldots \ldots$ & $\ldots \ldots \ldots$ & $\ldots \ldots \ldots$ & $\ldots \ldots \ldots \ldots$ & $\ldots \ldots \ldots \ldots$ & C....... & $\ldots$ TG. A. &. T.... \\
\hline$=36$ & $\ldots G \ldots \ldots$ & $\ldots \ldots \ldots$ & $\ldots \ldots \ldots$ & $\ldots \ldots \ldots$ & TA....... & $\ldots \ldots \ldots$ & . .TTG..A. & . . .... \\
\hline r37 & $\ldots G \ldots \ldots$ & $\ldots \ldots \ldots$ & $\ldots \ldots \ldots$ & $\ldots \ldots \ldots$ & TA....... & $\ldots \ldots$. & $\ldots$ TG.A. & . т.... \\
\hline$=38$ & $\ldots G \ldots \ldots$ & $\ldots \ldots \ldots$ & ...т. & $\ldots \ldots$ & $\ldots \ldots$ & C......G. & ...TG.TA. &. $\mathrm{T} \ldots \mathrm{C}$ \\
\hline 39 & $\ldots G \ldots \ldots$ & $\ldots$ & $\ldots \ldots$ & $\ldots$ & $\ldots$ & $\ldots \ldots$ TG & $\ldots$ TG. A. & . T.... \\
\hline 40 & $\ldots G \ldots \ldots$ & $\cdots$ & $\ldots \ldots \ldots$ & $\ldots \ldots \ldots$ & TA........ & $\ldots C \ldots G$ & T...TG. A. & $\ldots$ \\
\hline 41 & $\ldots$ G...T. & $\ldots \ldots \ldots \mathrm{T}$ & $\ldots \ldots \ldots c$ & $\ldots \ldots \ldots G$ & TA....... & $\ldots \ldots \ldots$ & ....TG. A. & $\ldots$ \\
\hline 42 & $\ldots G \ldots \ldots$ & $\cdots \cdots$ & $\ldots \ldots \ldots$ & $\ldots \ldots$ & TA. . . . . & $\ldots \ldots \ldots G$ & ...TG.A. & $\ldots$ \\
\hline 43 & .....T. & $\ldots \ldots \ldots$ & $\ldots \ldots \ldots$ & $\ldots \ldots \ldots$ & TA.C...G.. & $\ldots \ldots \ldots$ & . .TTG. . A. & $\ldots \ldots$ \\
\hline$\therefore 44$ & .G...T. & $\ldots \ldots \ldots$ & $\ldots \ldots \ldots$ & $\ldots \ldots \ldots \ldots$ & TA.C...G. . & $\ldots \ldots \ldots G$ & ....TG. . A. & $\ldots \ldots$ \\
\hline $\operatorname{Br} 45$ & $\ldots G \ldots \ldots$ & $\ldots \ldots \ldots$ & $\ldots \ldots \ldots$ & $\ldots \ldots \ldots$ & $\ldots \ldots \ldots$ & $\ldots \ldots G$ & ...TG. A. & $\ldots \ldots$ \\
\hline $\operatorname{Br} 46$ & $\ldots$ G...T. & $\ldots \ldots \ldots$ & $\ldots \ldots \ldots$ & $\ldots \ldots$.... & TA.C...G. & $\ldots \ldots \ldots$ G. & ....TG.A. & ...... \\
\hline $\operatorname{Br} 47$ & $\ldots \ldots \ldots$ & 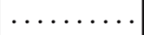 & $\ldots$ & $\ldots$ & $\ldots \ldots \ldots \ldots$ & $\ldots \ldots \ldots \ldots$ & $\ldots$ TG. . . & $\ldots$ т.... \\
\hline $\operatorname{Br} 48$ & $\ldots G \ldots \ldots$ & $-2+2$ & $\ldots \ldots$ & $\cdots$ & $\ldots \ldots \ldots$ & . & ...TTG.... & . T... \\
\hline $\operatorname{Br} 49$ & $\ldots$ G...T. & $\ldots \ldots \ldots \mathrm{T}$ & $\ldots$.....C & $\ldots \ldots$ & $\ldots \ldots$. . . . & $\ldots \ldots$ & $\ldots$ TG. A. & $\ldots \ldots c$ \\
\hline $\operatorname{Br} 50$ & $\ldots G \ldots \ldots$ & $\ldots$ & $\ldots \ldots$ & $\ldots$ & $\ldots \ldots \ldots$ & $\ldots \ldots \ldots$ & $\ldots$ TG.... & . т.... \\
\hline $\operatorname{Br} 51$ & $\ldots G \ldots$ & & $\ldots$ & $\ldots$ & TA... & $\ldots \ldots \ldots$ & ...TTG. A & $\ldots \ldots G$. \\
\hline BR52 & ..G... & & $\ldots$ & $\ldots$ & TA....... & $\ldots$. . ...G. & ...TG. A. & $\ldots \ldots$ \\
\hline & $\ldots G \ldots$ & $\ldots \ldots$ & $\ldots \ldots$ & $\ldots \ldots$ & $\ldots \ldots \ldots \ldots$ & $\ldots \ldots \ldots$ & ...TG.A. & ..... . \\
\hline & $\ldots \ldots \ldots$ & $\ldots \ldots$ & $\ldots \ldots$ & $\ldots \ldots$ & $\ldots \ldots \ldots$ & $\ldots \ldots \ldots$ & $\ldots$ TG $\ldots$ & $\ldots$ \\
\hline 55 & $\ldots G \ldots \ldots$ & $\cdots$ & $\ldots \ldots$ & $\ldots \ldots$ & TA...... & $\ldots \ldots \ldots$ G. & ...TTG..A. & . .т. \\
\hline 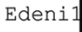 & …‥с & $\cdots$ & CC........ & .C.A.T.G. & TA.C...GTC &. CG... & TG. .TG..A. & тт... \\
\hline en & $\ldots$. T. C & $\cdots$ & CC........ & .C.A.T.G. & TA.C...GTC &. CG....... & T...TG.A. & TT..C... \\
\hline . & $\ldots \ldots$. & $\ldots \ldots \ldots{ }^{-}$ & CC....... & .C.A.T.G. & TA.C...GTC &. CG...... & TG. .TGA.A. & TT..... \\
\hline nura & T..CT.G.C. & .C.T. CTCGA & T. .GG.CAT. & CC.AATTG.. & ..C.GTA. C & $\ldots \ldots$ TGC & TC. TG.A. & TTT..G. \\
\hline i & .С...С.T. & .С.А....- &.$C C \ldots \ldots$ & .CGA..T..A & TA.C...G. & C...G...G. & T...TG..A. & С.т.... \\
\hline
\end{tabular}

Figure 3. Variable sites defining 55 mtDNA haplotype in B. brydei. The column on the left are haplotype ID. The numbers above are the nucleotide positions of the polymorphic sites starting from the 5' end of the mtDNA control region. Haplotypes ' 2 ' through '55' are listed with reference to haplotype ' 1 '. A dot indicates an identical nucleotide at the position relative to haplotype ' 1 '. Haplotypes of B. edeni, B. omurai and B. borealis published in Yoshida \& Kato (1999) and Wada et al. (2003) are included for comparative purposes / Sitios variables definiendo los 55 haplotipos de ADNmt in B. brydei. La columna sobre la izquierda son los ID de los haplotipos. Los números de arriba son las posiciones de nucleótidos de los sitios polimórficos a partir del extremo 5' de la región de control del ADNmt. Los haplotipos ' 2 ' a través de ' 55 ' se listan en referencia al haplotipo ' 1 '. Un punto indica un nucleótido idéntico en la posición relativa del haplotipo ' 1 '. Los haplotipos de $B$. edeni, B. omurai y B. borealis publicados en Yoshida \& Kato (1999) y Wada et al. (2003) se incluyen con fines comparativos 


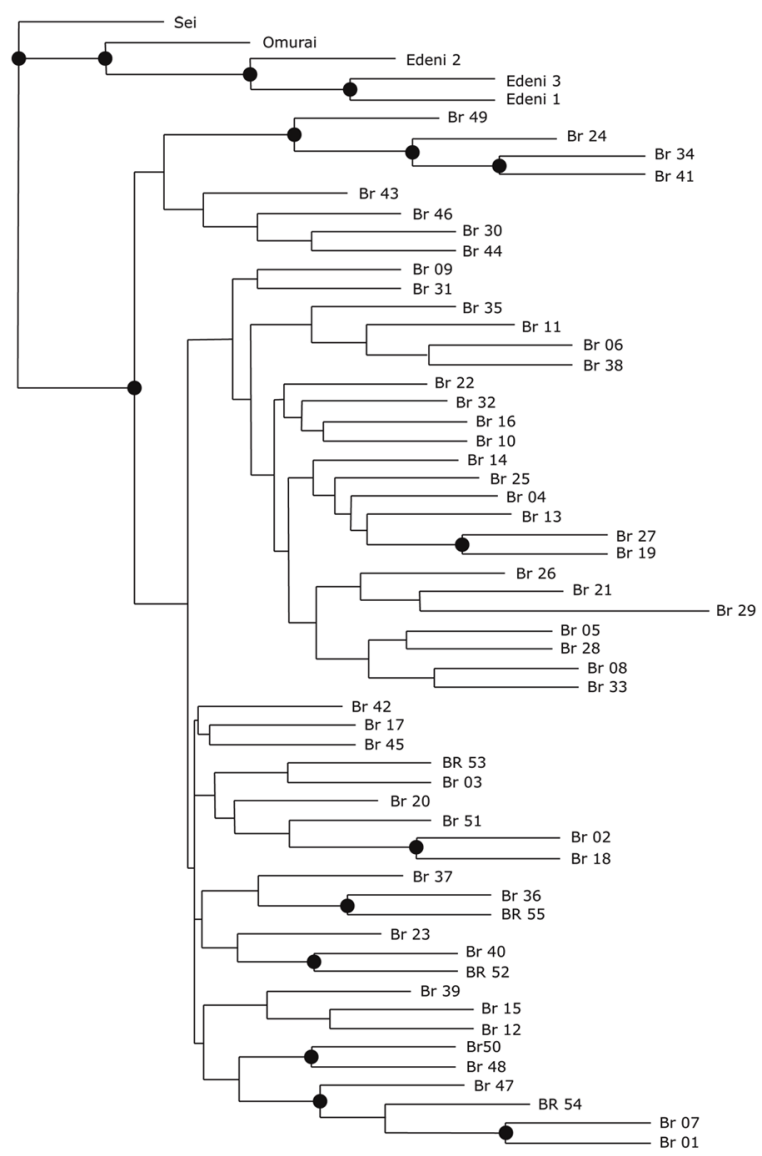

$-100$

Figure 4. Neighbor-Joining-based tree of Bryde's whale mtDNA haplotypes. Haplotypes were numbered following Kanda et al. (2007) (Br01, Br02, etc.) (see Table 2 and Fig. 3). Clades supported by over $50 \%$ in 1,000 bootstrap simulations are indicated by a dot. Note that haplotype 'Br36' corresponds to Wada et al. (2003)'s B. brydei haplotype / Árbol filogenético de los haplotipos de ADN mitochondrial generado por el método Neighbor-Joining. Haplotipos fueron numerados siguiendo a Kanda et al. (2007) (Br01, Br02, etc.) (ver Tabla 2 y Fig. 3). Clados apoyados por más de $50 \%$ en 1.000 simulaciones son indicados por un punto. Se debe notar que el haplotipo 'BR36' corresponde al haplotipo de B. brydei en Wada et al. (2003)

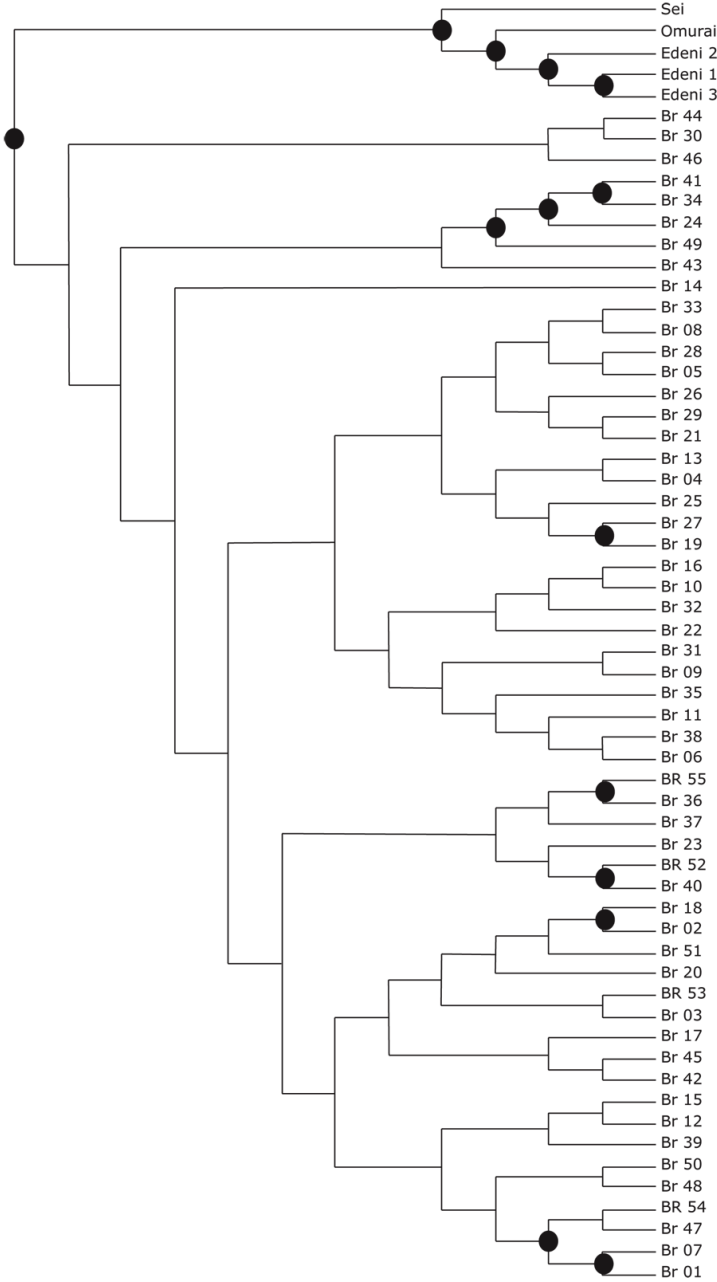

Figure 5. ML-based tree of Bryde's whale mtDNA haplotypes. Haplotypes were numbered following Kanda et al. (2007) (Br01, Br02, etc.) (see Table 2 and Fig. 3). Clades supported by over $50 \%$ in 1,000 bootstrap simulations are indicated by a dot / Árbol filogenético de los haplotipos de ADN mitochondrial generado por el método de ML. Haplotipos fueron numerados siguiendo a Kanda et al. (2007) (Br01, Br02, etc.) (ver Tabla 2 y Fig. 3). Clados apoyados por más de $50 \%$ en 1.000 simulaciones son indicados por un punto 


\section{GEOGRAPHICAL DISTRIBUTION OF B. BRYDEI HAPLOTYPES}

Among the 55 B. brydei haplotypes there was a total of 7 haplotypes in the Chilean sample, with 3 being specific to that locality. Four haplotypes (representing $70 \%$ of the total samples) were shared with the Peruvian sample. Only 2 haplotypes were discriminated in the Brazilian sample, one specific to that locality (one individual) and the other shared with all other localities except Java (7 individuals) (Table 2).

\section{GENETIC DIVERSITY}

Nucleotide diversity of $B$. bryde $i$ was similar among localities except in the case of Brazil where it was significantly lower (Table 3).

\section{PopUlation DIFFERENTIATION}

The comparison of dA between B. brydei from Peru and Chile yielded a negative value suggesting a close genetic relationship. However, the dA of B. brydei from Peru and Chile in relation to Brazilian whales was larger (Table 3).

There were not significant genetic differences between Chilean and Peruvian B. brydei. However both Chilean and Peruvian whales differed significantly from Brazilian whales. South American B. brydei differed significantly from all other populations of the Indian and Pacific Oceans (Table 4).

Table 3. Nucleotide diversity (on the diagonal) and Kimura's net-interpopulation genetic distance (dA) among populations of B. brydei. In parenthesis is the SE (WNP= western North Pacific) / Diversidad nucleotídica (sobre la diagonal) y distancia neta interpoblacional de Kimura $(\mathrm{dA})$ entre poblaciones de $B$. brydei. En paréntesis se denota la desviación estándar (WNP= Pacífico Noroccidental)

\begin{tabular}{lcccccc}
\hline & $\begin{array}{c}\text { WNP } \\
\mathrm{n}=401\end{array}$ & $\begin{array}{c}\text { Fiji } \\
\mathrm{n}=24\end{array}$ & $\begin{array}{c}\text { Peru } \\
\mathrm{n}=24\end{array}$ & $\begin{array}{c}\text { Chile } \\
\mathrm{n}=10\end{array}$ & $\begin{array}{c}\text { Brazil } \\
\mathrm{n}=8\end{array}$ & $\begin{array}{c}\text { Java } \\
\mathrm{n}=23\end{array}$ \\
\hline WNP & 0.0101 & 0.0012 & 0.0048 & 0.0058 & 0.0121 & 0.0115 \\
& $(0.0006)$ & & & & & \\
Fiji & & 0.0072 & 0.0069 & 0.0080 & 0.0126 & 0.0130 \\
Peru & & $(0.0018)$ & & & & \\
& & & $(0.0104$ & -0.0004 & 0.0060 & 0.0088 \\
Chile & & & & 0.0083 & 0.0057 & 0.0090 \\
Brazil & & & & $(0.0019)$ & 0.0008 & 0.0117 \\
Java & & & & & $(0.0006)$ & 0.0063 \\
& & & & & & $(0.0029)$ \\
\hline
\end{tabular}

Table 4. Results of the statistical test for genetic heterogeneity of B. brydei from South America (WNP= western North Pacific) / Resultados de las pruebas estadísticas de heterogeneidad genética de B. brydei de Sudamérica (WNP= Pacífico Noroccidental)

\begin{tabular}{lccc}
\hline & Hst & Kst* & Chi-square \\
\hline WNP-Peru & $0.0140(P=0.0001)$ & $0.0377(P=0.0001)$ & $P=0.0001$ \\
WNP-Chile & $0.0077(P=0.0001)$ & $0.0216(P=0.0001)$ & $P=0.0001$ \\
WNP-Brazil & $0.0214(P=0.0001)$ & $0.0379(P=0.0001)$ & $P=0.0001$ \\
Fiji-Peru & $0.1366(P=0.0001)$ & $0.2347(P=0.0001)$ & $P=0.0001$ \\
Fiji-Chile & $0.1275(P=0.0001)$ & $0.2522(P=0.0001)$ & $P=0.0001$ \\
Fiji-Brazil & $0.2630(P=0.0001)$ & $0.3964(P=0.0001)$ & $P=0.0001$ \\
Peru-Chile & $-0.0125(P=0.8535)$ & $-0.0163(P=0.9601)$ & $P=0.8939$ \\
Peru-Brazil & $0.1640(P=0.0001)$ & $0.2236(P=0.0001)$ & $P=0.0024$ \\
Chile-Brazil & $0.2195(P=0.0014)$ & $0.3323(P=0.0001)$ & $P=0.0039$ \\
Java-Peru & $0.2377(P=0.0001)$ & $0.3336(P=0.0001)$ & $P=0.0001$ \\
Java-Chile & $0.2511(P=0.0001)$ & $0.3514(P=0.0001)$ & $P=0.0001$ \\
Java-Brazil & $0.4836(P=0.0001)$ & $0.5292(P=0.0001)$ & $P=0.0001$ \\
\hline
\end{tabular}




\section{Discussion}

This paper presents the results of the first genetic analysis of South American Bryde's whales. Results suggested that whales from Peru, Chile and Brazil belong to B. brydei according to the taxonomic classification suggested by Wada et al. (2003).

The level of nucleotide diversity in the Brazilian $B$. bryde $i$ was significantly lower. To confirm this result, the analysis of additional samples from Brazil will be necessary. The current data set is limited to just 8 stranded animals, which could not be representative of the diversity of the population. Further analysis of additional samples, particularly from offshore areas, will be necessary to confirm the low level of genetic diversity in the Brazilian whales.

As expected, substantial genetic differentiation was found between eastern South Pacific and western South Atlantic B. brydei. This is consistent with the notion that $B$. bryde $i$ is distributed mainly north of approximately $40^{\circ} \mathrm{S}$ on both sides of South America, and that migration between the two ocean basins do not occur or that it is very limited. To evaluate the relative effects of divergence and migration between both sides of South America, the approach of Nielsen \& Wakeley (2001) should be used in future.

Populations separated by landmass have been reported for other species. For example common minke whale $B$. acutorostrata from both sides of the Japanese archipelago (Sea of Japan and western North Pacific) are genetically differentiated (Pastene et al. 2007). Genetic differentiation between animals in the west and east coast of South America has also been reported for other species such as the South American sea lion Otaria flavescens (Artico et al. 2010), humpback whale Megaptera novaeangliae (Engel et al. 2008) and dusky dolphins Lagenorhynchus obscurus (Cassens et al. 2003), which reveals a common phylogeographic pattern.

In contrast, $B$. brydei from Chile and Peru were closely related as suggested by a negative value of the net interpopulational distance and no statistically significant mtDNA differences between whales in these 2 localities. This result suggests that $B$. brydei from Chile and Peru belong to a same population. It should be recognized that the sample sizes used in the comparative genetic analysis between Peru and Chile's whales, were small, 24 and 10 samples, respectively. The analysis of a larger sample size is recommended to confirm this conclusion. However, if the effect size between the putative Peruvian and Chilean populations is at the same level as that between Chile and Brazil, then the small sample size used in this study should have been able to detect the difference, which it did not.

The sighting distribution data is consistent with the hypothesis of a north (Peru)-south (Chile) movement of B. brydei in the eastern South Pacific in spring and summer, which is consistent with our genetic results. Sightings of $B$. bryde $i$ have been related to a coastal upwelling ecosystem in central Chile in spring and summer (Gallardo et al. 1983). As the stomach contents of whales examined in central Chile were composed of pelagic fishes (unpublished information), the putative north-south migration in spring and summer could be related to food availability. Clearly further genetic studies based on samples from a broader longitudinal and latitudinal range are necessary to investigate additional structure within the western South Atlantic population.

Results of the genetic analyses provide no support for a differentiation of $B$. brydei in the eastern South Pacific as suggested by the IWC when it delineated a 'Peruvian stock' and an 'Eastern South Pacific stock' in the 80's (Donovan 1991), although the small number of samples in the present genetic analysis should be emphasized again. Genetic results presented here are consistent with the IWC delineation of a separated 'South Atlantic stock' (Donovan 1991), but it should be noted that the samples used in the present analysis covered only a small portion of the South Atlantic (southern and south eastern coast of Brazil) and the analyses of additional samples from broader geographical areas is necessary to investigate additional structure in this population as mentioned above.

In summary, no significant genetic differences were found between B. brydei of Chile and Peru. B. brydei from Peru and Chile were genetically different from whales from Brazil. B. brydei from all South American localities were genetically different from whales from other localities of the Pacific Ocean and Indian Ocean confirming that this species is highly structured within and between ocean basins. The genetic differences among populations were smaller within an oceanic basin than between oceanic basins. Furthermore the level of genetic differentiation, especially at the inter-equatorial level, was smaller than that of common minke whale (Pastene et al. 2007, 2010) and humpback whale (Baker et al. 1994), which could be due to their more recent separation compared to other baleen whale species (Kanda et al. 2007). 


\section{ACKNOWLEDGMENTS}

We thank M. Goto, Institute of Cetacean Research, for his assistance in the analysis of data. We also thank the researchers from GEMARS for collaborating in the collection and necropsies of the specimens along the Brazilian coast. We are also grateful to the respective Directorships of Fundación CEQUA and Instituto Antártico Chileno for providing time for preparation of the manuscript (CONICYT Regional Grant number R13A1002), and Inti González of CEQUA for the preparation of map in SIG. J.F. Moura gratefully acknowledge CAPES and the Alexander von Humboldt Foundation for financial support (Proc. BEX 0128/14-7).

\section{LITERATURE CITED}

Aguayo A, R Bernal, C Olavarria, V Vallejos \& R Hucke. 1998. Observaciones de cetáceos realizadas entre Valparaíso e Isla de Pascua, Chile, durante los inviernos de 1993, 1994 y 1995. Revista de Biologia Marina y Oceanografia 33(1): 101-123.

Andriolo A, JM da Rocha, AN Zerbini, PC Simões-Lopes, IB Moreno, A Lucena, D Danilewicz \& M Bassoi. 2010. Distribution and relative abundance of large whales in a former whaling ground off eastern South America. Zoologia 27(5): 741-750.

Arnason U, A Gullberg \& B Widegren. 1993. Cetacean mitochondrial DNA control region: sequences of all extant baleen whales and two sperm whale species. Journal of Molecular Biology and Evolution 70: 960-970.

Artico LO, A Bianchini, KS Grubel, DS Monteiro, SC Estima, LR de Oliveira, SL Bonatto \& LF Marins. 2010. Mitochondrial control region haplotypes of the South American sea lion Otariaflavescens (Shaw, 1800). Brazilian Journal of Medical and Biological Research 43(9): 816820.

Baker CS, RW Slade, JL Bannister, RB Abernethy, MT Weinrich, J Lien, J Urban-Ramirez, P Corkeron, J Calambokidis, O Vasquez \& SR Palumbi. 1994. Hierarchical structure of mitochondrial DNA gene flow among humpback whales, world-wide. Molecular Ecology 3: 313-327.

Bastida R \& D Rodriguez. 2009. Marine mammals of Patagonia and Antarctica, 208 pp. Vasquez Mazzini Editores, Buenos Aires.

Cassens I, K Van Waerebeek, PB Best, EA Crespo, J Reyes \& MC Milinkovitch. 2003. The phylogeography of dusky dolphins (Lagenorhynchus obscurus): a critical examination of network methods and rooting procedures. Molecular Ecology 12: 1781-1792.

Clarke R \& A Aguayo. 1965. Bryde's whale in the south-east Pacific. Norsk Hvalfangsttid 54(7): 141-148.
Donovan GP. 1991. A review of IWC stock boundaries. Report of the International Whaling Commission, Special Issue 13: $39-68$.

Engel MH, NJR Fagundes, HC Rosenbaum, MS Leslie, PH Ott, R Schmitt, E Secchi, L Dalla Rosa \& SL Bonatto. 2008. Mitochondrial DNA diversity of the Southwestern Atlantic humpback whale (Megaptera novaeangliae) breeding area off Brazil, and the potential connections to Antarctic feeding areas. Conservation Genetics 9: 1253-1262.

Felsenstein J. 1993. PHYLIP (Phylogenny Inference Package). 3.5c. University of Washington, Seattle.

Figueiredo LD, RH Tardin, L Lodi, ISI Maciel, MAS Alves \& SM Simão. 2014. Site fidelity of Bryde's whales (Balaenoptera edeni) in Cabo Frio Region, Southeastern Brazil, through photoidentification technique. Brazilian Journal of Aquatic Science and Technology 18(2): 59-64.

Gallardo VA, D Arcos, M Salamanca \& LA Pastene. 1983. On the occurrence of Bryde's whale (Balaenoptera edeni Anderson 1878) in an upwelling area off Central Chile. Report of the International Whaling Commission 33: 481-488.

Hudson RR, BB Boos \& NL Kaplan. 1992. A statistical test for detecting geographic subdivision. Molecular Biology and Evolution 9: 138-151.

Kanda N, M Goto, H Kato, MV Mcphee \& LA Pastene. 2007. Population genetic structure of Bryde's whales (Balaenoptera brydei) at the inter-oceanic and transequatorial levels. Conservation Genetics 8: 853-864.

Kershaw F, MS Leslie, T Collins, RM Mansur, BD Smith, G Minton, R Baldwin, RG LeDuc, RC Anderson, RL Jr Brownell \& HC Rosenbaum. 2013. Population differentiation of 2 forms of Bryde's whales in the Indian and Pacific Oceans. Journal of Heredity 104(6): 755-764.

Kimura M. 1980. A simple method for estimating evolutionary rates of base substitutions through comparative studies of nucleotide sequences. Journal of Molecular Evolution 16: 111-120.

Krützen M, LM Barré, LM Möller, MR Heithaus, C Simms \& WB Sherwin. 2002. A biopsy system for small cetaceans: darting success and wound healing in Tursiops spp. Marine Mammal Science 18: 863-878.

Maddison DR \& WP Maddison. 2000. Analysis of phylogeny and character evolution, 492 pp. Sinauer Associates, Sunderland.

Nei M. 1987. Molecular evolutionary genetics, 512 pp. Columbia University Press, New York.

Nei M \& WH Li. 1979. Mathematical model for studying genetic variation in terms of restriction endonucleases. Proceedings of the National Academy of Science, USA 76: 5269-5273.

Nielsen R \& J Wakeley. 2001. Distinguishing migration from isolation. A Markov chain Monte Carlo approach. Genetics 158: 885-896. 
Pastene LA. 1982. Análisis de las capturas de ballenas efectuadas por la industria ballenera nacional en el sector del Pacifico Sur Oriental correspondiente a Chile y consideraciones del estado actual de dicha industria y su desarrollo histórico. Tesis de Biólogo Marino, Facultad de Ciencias Biológicas y de Recursos Naturales, Universidad de Concepción, Concepción, 79 pp.

Pastene LA, M Goto, N Kanda, AN Zerbini, D Kerem, K Watanabe, Y Bessho, M Hasegawa, R Nielsen, F Larsen \& PJ Palsbøll. 2007. Radiation and speciation of pelagic organisms during periods of global warming: the case of the common minke whale, Balaenoptera acutorostrata. Molecular Ecology 16: 1481-1500.

Pastene LA, J Acevedo, M Goto, AN Zerbini, P Acuña \& A Aguayo-Lobo. 2010. Population structure and possible migratory links of common minke whales, Balaenoptera acutorostrata, in the Southern Hemisphere. Conservation Genetics 11: 1553-1558.

Rice DW. 1998. Marine mammals of the world: systematics and distribution, Special Publication 4: 234 pp. The Society for Marine Mammalogy, Allen Press, Kansas

Saitou N \& M Nei. 1987. The neighbor-joining method: a new method for reconstructing phylogenetic tree. Molecular Biology and Evolution 4(4): 406-425.

Sambrook J \& DW Russell. 2001. Molecular cloning. A laboratory manual, 2344 pp. Cold Spring Harbor Laboratory Press, Cold Spring Harbor.
Siciliano S, MCO Santos, AFC Vicente, E Zampirolli, JL Brito Jr, AF Azevedo \& JLA Pizzorno. 2004. Stranding and feeding records of Bryde's whales (Balaenoptera edeni) in south-eastern Brazil. Journal of the Marine Biological Association of the United Kingdom 84: 857-859.

Tamura K \& M Nei. 1993. Estimation of the number of nucleotide substitutions in the control region of mitochondrial DNA in humans and chimpanzees. Molecular Biology and Evolution 10: 512-526.

Tamura K, G Stecher, D Peterson, A Filipski \& S Kumar. 2013. MEGA6: Molecular evolutionary genetics analysis version 6.0. Molecular Biology and Evolution 30: 27252729.

Valdivia J, P Ramirez \& F Franco. 1981. The exploitation of Bryde's whales in the Peruvian Sea. Report of the International Whaling Commission 31: 441-448.

Wada S, M Oishi \& T Yamada. 2003. A newly discovered species of living baleen whale. Nature 426: 278-281.

Yoshida H \& H Kato. 1999. Phylogenetic relationships of Bryde's whales in the western North Pacific and adjacent waters inferred from mitochondrial DNA sequences. Marine Mammal Science 15: 1269-1286.

Zerbini AN, ER Secchi, S Siciliano \& PC Simões-Lopes. 1997. A review of the occurrence and distribution of whales of the Genus Balaenoptera along the Brazilian coast. Report of the International Whaling Commission 47: 407-417.

Recibido el 5 de septiembre de 2014 y aceptado el 11 de junio de 2015

Editor Asociado: Maritza Sepúlveda M. 